\title{
Age and type of aphasia in patients with stroke
}

\author{
P J ESLINGER, AND A R DAMASIO
}

\author{
From the Department of Neurology (Division of Behavioral Neurology \& Benton Laboratory of \\ Neuropsychology) University of Iowa College of Medicine, Iowa City, USA
}

SUMMARY The age and gender of a series of patients with different types of aphasia were analysed. Regardless of gender, patients with Broca and conduction aphasias were significantly younger than those with Wernicke and global aphasias. Considering the established cerebral localisation of each of those aphasia types, it appears that, with age, stroke in the territory of the middle cerebral artery will tend to either shift posteriorly (producing Wernicke aphasia) or occupy most of the middle cerebral artery territory (producing global aphasia). But in the absence of concurrent verification of the locus of lesion in each of the cases in our sample, a possible alternative hypothesis must be entertained: that there might be age-related changes in the neurophysiological mechanism subserving language, such that some types of aphasia would tend to be more prevalent with age, regardless of lesion location.

Some neurologists with experience in the field of aphasia have held the belief that stroke patients tend to exhibit different aphasic syndromes at different ages. In a recent study, Obler, Albert, Goodglass and Benson ${ }^{1}$ have gathered evidence in support of such an impression. In two other studies dealing with aphasia and stroke, although not aimed at the question of age and aphasia type, the available data hinted at similar differences in aphasia type and its relation to age. ${ }^{2} 3$

In the study of right-handed males by Obler et al, ${ }^{1}$ the median (Md) age of the aphasic patients was 56 years $($ mean $=56 \cdot 1)$. Broca aphasics appeared to be the youngest age group $(\mathrm{Md}=51)$ and Wernicke aphasics the oldest $(\mathrm{Md}=63)$. The median age for other aphasia types varied between those extremes (conduction $\mathrm{Md}=57 \cdot 5$; global $\mathrm{Md}=56$; anomic $M d=54)$. In the study by Kertesz et al, ${ }^{2}$ whose purpose was the investigation of radioisotope correlates of aphasia produced by stroke, the mean group age for Broca aphasics was 55.1 years. Wernicke, conduction, anomic and global aphasics ranged between $64 \cdot 5-65 \cdot 7$ years. Surprisingly, statistical analysis did not reveal significant group differences in relation to age. Finally, the observations of Brust et al, ${ }^{3}$ also revealed that Broca aphasics were generally the youngest among stroke patients. However, when Broca and Anomic aphasics were combined to form a nonfluent aphasic group, there were no significant differences from fluent aphasics.

Address for reprint requests: AR Damasio, M.D. Department of Neurology University Hospitals Iowa City, IA 52242, USA.

Accepted 22 January 1981
In the latter two studies, the composition of groups with respect to factors such as gender and handedness was not reported, leaving the possibility open that intergroup variability might have been related to factors other than age. In spite of that fact, the data suggest that a relationship may exist between age and aphasia type. The following study was designed to ascertain whether a similar relationship might be present in the population of aphasic patients studied in our institution. Should it be present we intended to determine if gender, in addition to age, might account for the interaction, and if any predictive associations might exist that would have diagnostic value. Finally, we wished to discuss possible explanations for such a relationship.

\section{Methods and materials}

Case histories of aphasic patients were obtained from a retrospective search of records of the Department of Neurology (Division of Behavioral Neurology) at the University of Iowa College of Medicine. Patients were selected according to the following criteria: (1) righthandedness, (2) established documentation of single, unilateral infarction consequent to embolus, thrombus, or haemorrhage, (3) unequivocal diagnosis of aphasia obtained with the same diagnostic instrument (Multilingual Aphasia Examination ${ }^{4}$, and (4) classification according to the same criteria. ${ }^{5}$ Owing to the strictness of the selection many patients could not be included, but a total of 64 cases met the criteria. For each case, the following data were noted: (a) age at time of infarction, (b) type of aphasia, and (c) gender. Thirty-six were males and 28 were females. The aphasia types were 11 Broca, 
9 conduction, 27 Wernicke and 17 global aphasics. There were seven patients with transcortical aphasias (five transcortical sensory and two transcortical motor) who were not included in the statistical analyses. However, the transcortical sensory aphasics are represented in the figures for purposes of comparison. Data analysis included the median test to assess similarity of age distributions among aphasic groups, and the Mann-Whitney $U$ test and the Pearson chi square test to investigate gender differences and predictive association among the variables of age, gender and type of aphasia.

\section{Results}

Summary data for each aphasic group are presented in table 1 and age distributions are outlined in table 2. Formal analysis of age differences in aphasia was undertaken on the combined data of male and female aphasics. As presented in table 1, median ages varied widely among aphasic groups. This observation was supported by statistical analysis $\left(\chi^{2}=14 \cdot 169, \mathrm{df}=3\right.$, $\mathrm{p}<0.005)$ which indicated a significant difference in age distributions (see figures 1 and 2). More powerful and specific comparisons were carried out to determine exactly where the differences occurred. MannWhitney $U$ tests for each pair of aphasic groups revealed that Broca and conduction aphasics did not differ $(U=33, p>0.05)$ although each was significantly different from both global and Wernicke aphasic groups ( $p<0.001$ in each case). The difference

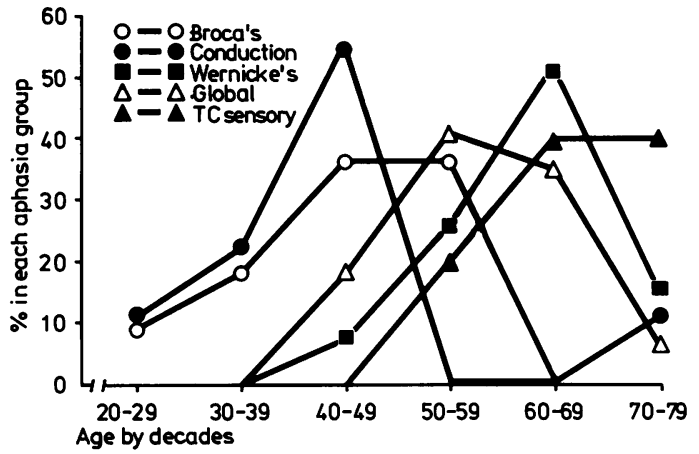

Fig 1 Age distributions of aphasia groups.

between global and Wernicke aphasics approached but did not reach significance in a two-tailed test $(\mathrm{U}=159 \cdot 5,0.05<\mathrm{p}<0.10)$.

Since these results were obtained from combined groups of male and female aphasics, the role of gender was investigated in two ways. First, analysis of all males and females, regardless of aphasia diagnosis, revealed no significant gender differences in age $(z=1.02, p=0.846)$. Secondly, assessment of gender differences in age within each aphasia type was undertaken. Again, no differences attributable to gender were evident ( $p>0.05$ in each case).

Table 1 Age summary for aphasia types

\begin{tabular}{|c|c|c|c|c|c|c|c|c|c|}
\hline & \multicolumn{3}{|c|}{ All aphasics } & \multicolumn{3}{|c|}{ Males } & \multicolumn{3}{|c|}{ Females } \\
\hline & $n$ & Mean & Median & $n$ & Mean & Median & $n$ & Mean & Median \\
\hline $\begin{array}{l}\text { Broca } \\
\text { Conduction } \\
\text { Wernicke } \\
\text { Global } \\
\text { Overall (years) }\end{array}$ & $\begin{array}{r}11 \\
9 \\
27 \\
17 \\
64\end{array}$ & $\begin{array}{l}44 \cdot 5 \\
42 \cdot 9 \\
62 \cdot 1 \\
58 \\
55 \cdot 3\end{array}$ & $\begin{array}{l}46 \\
43 \\
63 \\
58 \\
57\end{array}$ & $\begin{array}{r}3 \\
4 \\
18 \\
11 \\
36\end{array}$ & $\begin{array}{l}48 \cdot 3 \\
46 \\
61 \\
56 \cdot 4 \\
56 \cdot 9\end{array}$ & $\begin{array}{l}48 \\
42 \cdot 5 \\
60 \\
55 \\
57 \cdot 5\end{array}$ & $\begin{array}{r}8 \\
5 \\
9 \\
6 \\
28\end{array}$ & $\begin{array}{l}43 \\
40 \cdot 4 \\
64 \\
61 \\
53 \cdot 2\end{array}$ & $\begin{array}{l}44 \cdot 5 \\
43 \\
63 \\
61 \cdot 5 \\
56 \cdot 5\end{array}$ \\
\hline
\end{tabular}

Table 2 Age distribution of aphasic types according to age and gender

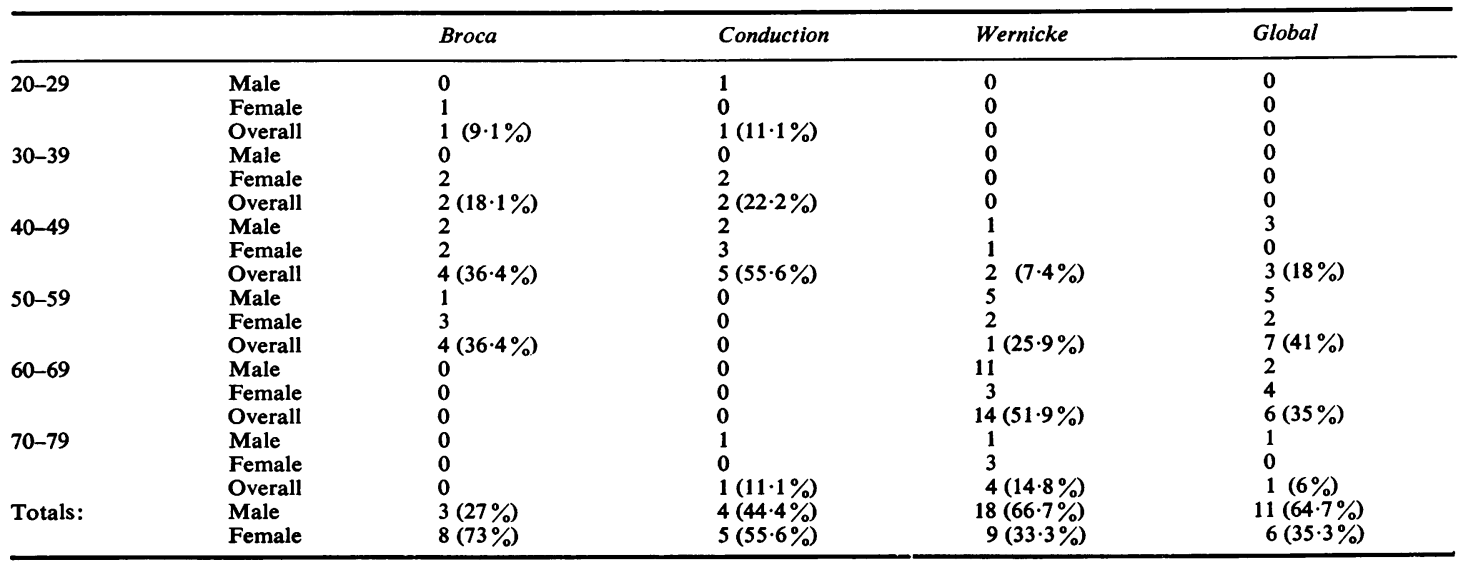




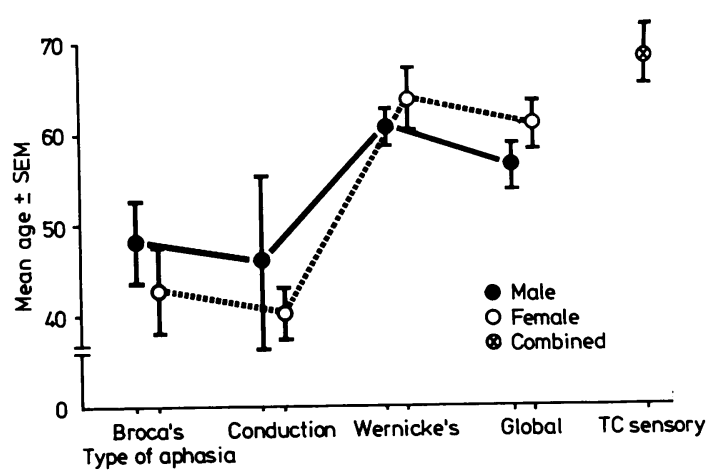

Fig 2 Mean age $\pm S E M$ of aphasia groups according to gender.

Because of the clear predominance of males among Wernicke and global aphasia types, further analysis was directed toward determining whether the prevalence of males and females was statistically associated with aphasia type. A Pearson chi square test of association was found to be significant $\left(\chi^{2}=10.94\right.$, $\mathrm{df}=3, \mathrm{p}<0.05$ ), indicating that some association does exist between the prevalence of gender types and aphasia type. It is clear that females predominated among Broca aphasics while males predominated among Wernicke and global aphasics (see table 2).

In exploring the relationships among aphasia type, aging, and gender, we were interested in determining whether predictive associations might exist. Such a possibility was investigated by calculating indices of predictive association. In a prediction of gender, knowledge of aphasia type reduces the probability of error by $21.4 \%$ on the average. This accords well with our earlier finding that some degree of association does exist between gender and type of aphasia. Interestingly, there is no clear reduction in error for a prediction of aphasia type derived from knowledge of gender alone. On the other hand, information regarding age can help the prediction of aphasia type by reducing the probability of error by $10.8 \%$ on the average. Conversely, knowledge about aphasia type reduces the probability of error in predicting age by an average of $28 \%$. Moreover, by knowing either age or aphasia type, the predictive ability of the other can be improved by an average of $17 \cdot 5 \%$.

\section{Discussion}

Three basic facts emerged from this investigation. Firstly, age differences were found among aphasia types; specifically, Broca and conduction aphasias while not differing, occurred more frequently at younger ages than both Wernicke and global aphasias. Although Wernicke aphasics composed the oldest group, they were not different from global aphasics. These results are largely in agreement with those of Obler et al, ${ }^{1}$ and suggest that althought any aphasia type may be found at virtually any age, a young stroke patient has a higher chance of developing Broca or conduction aphasia than Wernicke, global or transcortical sensory aphasia. The converse is true for an elderly stroke patient. Secondly, the age differences among aphasia types could not be attributed to gender, since the ages of males and females were not different. However, probing of the data revealed that prevalence of males and females was not independent of aphasia type, suggesting some form of association. There were more male than female aphasics overall and, interestingly, almost double the number of males among Wernicke and global aphasics. This is consistent with epidemiological data demonstrating a higher prevalence of males among all stroke patients as well as with increasing age. ${ }^{6}$ Thirdly, the computation of indices of predictive association indicated that the probability of error in predicting (a) gender from knowledge of aphasia type, and in predicting (b) either age or aphasia type from knowledge of the other, could be reduced. These associations, however, did not offer precision of prediction for two reasons: first, both males and females were found in each type of aphasia, and second, there was clustering of aphasia types in relation to age, that is, Broca-conduction and Wernicke-global, rather than discriminative intervals between types. Thus, age and gender may account for only a part of the variance associated with aphasia type. Furthermore, conclusions from these data must be tempered by the limited sample of aphasic patients and aphasia types. It should be noted that case material was restricted in an effort to ensure an homogeneous sample of stroke patients. Thus, our conclusions should only apply to similar case material.

What could be the basis for the relationship between age and type of aphasia? Several hypotheses have been formulated:1 78 (1) there might be some neuropathological variation associated with increasing age, such that the preferred locus of infarction would shift to posterior brain sites; consequently, younger patients would demonstrate a higher incidence of relatively anterior strokes and older patients would exhibit predominantly posterior strokes; (2) there might be changes in cerebral blood flow associated with aging, predisposing different brain areas to stroke; (3) the higher incidence of Wernicke's aphasia in older patients might be related to the cumulative effects of mental decline associated with aging and insult to language areas of the brain, regardless of its anatomical locus; (4) 
there might be continuous, age-related changes in the neurophysiological mechanisms subserving language functions, such that regardless of lesion location certain aphasia types might become more prevalent with age; finally, (5) older patients might not recover from comprehension defects as readily as younger patients, thereby leading to a prevalence of Wernicke's aphasia.

The first two hypotheses, regarding gross neuropathological variations associated with aging and cerebral blood flow changes, are not mutually exclusive. In fact, decreased cerebral blood flow in a specific area could account for gross pathological changes in that area, so that hypothesis (2) is actually a component of (1). This is an intriguing and testable possibility and our own view is that (a) the relation between age and aphasia type is possibly a relation between age and locus of cerebral infarction within the perisylvian region; and that (b) a parsimonious explanation for the association between lower age and anterior infarction and higher age and posterior infarction, resides in the vascular system of the area. In short, we suggest that the prevalence of Wernicke, transcortical sensory and global aphasias, with age, is due to the increased prevalence, with age, of middle cerebral artery strokes which either shift posteriorly or occupy a larger portion of the middle cerebral artery territory. Support for our position is as follows: (a) the relationship between type of aphasia and locus of lesion has been well documented and it appears to hold for any age in the adult range; in fact, the discovery of a link between anterior and posterior loci for the aphasias derived from the study of many elderly patients affected by stroke, (b) recent neuroradiological studies 2910 have demonstrated that the major aphasia syndromes have considerably distinct anatomical loci, varying along the anterior-posterior axis of the perisylvian region, (c) the fact that infarctions which produce Wernicke or transcortical sensory aphasias are located in the terminal branches of the middle cerebral artery, while those which produce Broca and conduction aphasias are related to the early branches of the same artery, raise questions regarding the vulnerability to age of those different vascular beds; for example, age might render the terminal branches of the middle cerebral artery more vulnerable to infarction than the early branches, (d) the age curve of patients with Wernicke aphasia was parallel to that of transcortical sensory aphasia (fig 1), a rather interesting finding given the anatomical and behavioural contiguity of both syndromes.

Let us now consider the other three hypotheses regarding age and type of aphasia. A possibly valid one (4) suggests that, irrespective of lesion location, different aphasia syndromes would be more prevalent at different ages. The basis for such differential prevalence would be found in age-related changes in the neurophysiological mechanism subserving language. Wernicke, transcortical sensory and global aphasias could become slightly more frequent in old age. Leaving aside its relative arbitrariness, the main obstacle to this hypothesis is anatomical: in an adult population composed of right handers with left language dominance, there is no evidence to suggest that the locus of damage resulting in Wernicke aphasia is anything but posterior, regardless of age, just as there is no evidence that the loci of damage producing Broca and conduction aphasias are anything but anterior, regardless of age (the only known exceptions being young children, some lefthanders and exceptional right-handers). Yet the hypothesis deserves attention and so these anatomical relationships should be subjected to further empirical analysis by study of CT scans in young and old patients with aphasia.

Hypothesis (3) appears invalid. Patients with Wernicke aphasia can be demented but clearly are not, in most cases. Their non-verbal cognitive functioning, which is the principal psychometric indicator of mental decline, often remains intact. Thus, while it is possible that dementia may be observed more frequently in Wernicke aphasics than in Broca aphasics, there is no evidence to the effect that dementia is a necessary or predisposing condition for the appearance of posterior fluent aphasias. As for hypothesis (5), its scientific basis remains obscure. To suggest that rate of recovery is the critical factor separating younger Broca and older Wernicke aphasics is tantamount to hypothesising that younger patients develop Wernicke aphasia initially but that recovery of comprehension will remove them from the Wernicke aphasia category. The unlikely implication is that such syndromes develop without damage to Wernicke's area, given the speedy recovery of language comprehension.

Our findings and the questions raised by them pertain more to the physiopathology of stroke than to the physiopathology of aphasia. Accordingly, further research is in progress to examine the relation between age and locus of lesion and to probe its causes, using a criterion other than the presence of aphasia.

\section{References}

1 Obler LK, Albert ML, Goodglass H, Benson DF. Aphasia type and aging. Brain Lang 1978; 6:318-22.

2 Kertesz A, Lesk D, McCabe P. Isotope localization of infarcts in aphasia. Arch Neurol 1977; 34:590-601.

3 Brust JC, Shafer SQ, Richter RN, Bruun B. Aphasia in acute stroke. Stroke 1976; 7:167-74. 
4 Benton, AL. Multilingual Aphasia Examination. Department of Neurology, University of Iowa College of Medicine, 1976.

5 Goodglass H, Kaplan E. The Assessment of Aphasia and Related Disorders Philadelphia: Lea and Febiger, 1972.

6 Sahs AL, Hartman EC, eds. Fundamentals of Stroke Care, DHEW Publication No. (HRA) 76-14016, 1976.

7 Benson DF. Age, aphasia, and stroke localization.
Arch Neurol 1978; 35:619-20.

8 Carter JE. Age, aphasia, and stroke localization. Arch Neurol 1978; 35:619.

9 Benson DF, Patten DH. The use of radioactive isotopes in the localization of aphasia-producing lesions. Cortex 1967; 3:258-71.

10 Damasio H, Damasio A, Hamsher K, Varney $\mathrm{N}$. CT scan correlates of aphasia and allied disorders. Neurology (Minneap) 1979; 29:572. 